\title{
Erratum
}

\section{Erratum to: Analytical Solutions of Cracks Emanating from an Elliptic Hole in an Infinite Plate under Tension}

LIU Shuhong ${ }^{1,2, *}$ and DUAN Shijie ${ }^{3}$

1 Department of Engineering Mechanics, Shijiazhuang Tiedao University, Shijiazhuang 050043, China

2 The Key Laboratory for Health Monitoring and Control of Large Structures of Hebei Province,

Shijiazhuang 050043, China

3 Department of Adult Education, Shijiazhuang Tiedao University, Shijiazhuang 050043, China

The original version of this article unfortunately contained a mistake. The presentation of the DOI in printed version was incorrect. The corrected DOI is given below.

DOI: 10.3901/CJME.2014.0526.300

* Corresponding author. E-mail: liush@stdu.edu.cn

Supported by Hebei Provincial Natural Science Foundation of

China(Grant No. A2011210033), and Foundation of Hebei Education

Department of China(Grant No. ZH2011116)

(C) Chinese Mechanical Engineering Society and Springer-Verlag Berlin Heidelberg 2014 